\title{
Numerical Study on Liquid Fuel Spray Behavior in Turbulent Flames Using LES*
}

\author{
Yuichi ITOH $^{* *}$ and Nobuyuki OSHIMA*** \\ ** Department of Mechanical Engineering, Kisarazu National College of Technology \\ 2-11-1 Kiyomidai-Higashi, Kisarazu, Chiba 292-0041, Japan \\ E-mail: itoh@m.kisarazu.ac.jp \\ *** Division of Mechanical \& Space Engineering, Graduated School of Engineering, Hokkaido University \\ Kita-13 Nishi-8 Kitaku, Sapporo, Hokkaido 060-8628, Japan
}

\begin{abstract}
In the present study, Large-Eddy Simulation (LES) modeling for turbulent spray combustion flows has been developed in conjunction with the Eulerian/Lagrangian method for the gas phase and dispersed phase representation and flamelet approach for combustion modeling. Moreover, a new thermal energy coupling model is proposed to estimate the thermal interaction between the gas phase and the dispersed phase. The governing equations for the gas phase are the mass and momentum conservation equations and the mixture fraction transport equation. The governing equations for spray droplets are the mass, momentum, and thermal energy equations for each droplet. We applied the numerical procedure to a laboratory-sized spray combustor and found that procedure can predict key features of turbulent spray combustion phenomena, such as the temperature distribution of the combustion gas, individual droplet behavior, and droplet vaporization phenomena. Moreover, the flame temperature drop caused by the thermal impact from the spray droplets is expressed properly by employing the coupling model.
\end{abstract}

Key words : Large-Eddy Simulation, Spray Flame, Droplet Vaporization

\section{Nomenclature}

$c_{p}:$ Specific heat

$D$ : Droplet diameter, Typical length

(burner-tip diameter)

$g$ : Gravity

$h:$ Heat transfer coefficient

$m:$ Mass

$p:$ Pressure

$T:$ Temperature

$u_{i}$ : Velocity component

$Y$ : Mass fraction

\section{1. subscript}

$$
g: \text { gas phase value }
$$

$d$ : dispersed phase value

\author{
$Z:$ Mixture fraction \\ $\mathrm{Nu}:$ Nusselt number \\ Re : Reynolds number \\ Sc : Schmidt number \\ $\mathrm{Sh}:$ Sherwood number \\ $\delta_{i j}:$ Kronecker delta \\ $\lambda$ : Thermal conductivity \\ $\mu$ : Dynamic viscosity \\ $\rho$ : Density
}

\section{2. superscript}

$k:$ droplet number

\section{Introduction}

Spray combustion is frequently used for industrial combustion systems such as diesel

engines, furnaces, and gas-turbine combustors, because it has several good characteristics for 
realizing high-loaded combustion and high response. Strict environmental regulations require us to develop combustor systems with higher efficiency, higher performance, and lower emissions. As a result, design optimization of these systems has been investigated experimentally and numerically.

Numerical simulations of spray combustion are currently carried out using a steady-state approach such as the Reynolds-averaged Navier-Stokes approach (RANS) ${ }^{(1),(2)}$. Previous studies have shown reasonable progress in the time-averaged results. However, these models have a limited ability to predict unsteady features in turbulent spray combustion flows such as blow off, ignition, and representation for dispersed droplets. On the other hand, Large-Eddy Simulation (LES) achieved good prediction for turbulent unsteady flow field, e.g., Ref. (3), particle laden flows ${ }^{(4),(5)}$, turbulent combustion flows ${ }^{(6),(7)}$, and turbulent flow with vaporizing spray droplets $^{(8)}$.

Although last few years several challenges have been made on computation of $\operatorname{LES}^{(9),(10)}$ and $\operatorname{DNS}^{(11),(12)}$ for turbulent spray combustion system, comparison and validation with experimental data is not yet enough.

In the present study, these droplet and turbulent combustion models are extended and applied to the numerical analysis of turbulent spray combustion flow to develop an accurate simulation code that can predict physical quantities correctly in turbulent spray combustion flows using Large-eddy simulation. Moreover, a new thermal energy coupling model is proposed to estimate the thermal interaction between the gas phase and the dispersed phase.

\section{Numerical Procedure}

The governing equations for the gas phase are described in an Euler representation, while the fuel droplet motion equations are described in a Lagrangian representation. In our computation, it is assumed that the droplet diameters are sufficiently small, the number density of the droplets is dilute, and influences of the droplets on the flow field can be neglected. Thus, gas-phase motion and droplet motion can be solved separately ${ }^{(13)}$.

\subsection{Governing Equations for Gas Phase}

The governing equations for the gas phase are the mass and momentum conservation equations and the mixture fraction transport equation. These equations are discretized in a cylindrical coordinate system. In our computation, LES is employed to solve the gas-phase equations. In LES calculation, resolved (grid) scales are directly calculated, while unresolved (sub-grid) scales are modeled. Thus, the flow variables are decomposed into resolved and sub-grid scale components as follows:

$$
f=\bar{f}+f^{\prime}
$$

where the overbar $\left({ }^{-}\right)$means a spatially filtered value representing a resolved scale, and $\left(^{\prime}\right)$ indicates its fluctuation component representing the sub-grid scale. For variable-density flows such as turbulent combustion flows, the basic LES equations are modified using a densityweighted (Favre) filter, as follows:

$$
\begin{aligned}
& f=\widetilde{f}+f^{\prime \prime} \\
& \widetilde{f} \equiv \frac{\overline{\rho f}}{\bar{\rho}}
\end{aligned}
$$

where the tilde $(\sim)$ represents a Favre-filtered value. Applying the filter operations to the governing equations for flow fields, the following equations are derived:

$$
\frac{\partial \bar{\rho}}{\partial t}+\frac{\partial \bar{\rho} \widetilde{u}_{j}}{\partial x_{j}}=\bar{\psi}_{v}
$$




$$
\begin{aligned}
\frac{\partial \widetilde{\rho} \widetilde{u}_{i}}{\partial t}+\frac{\partial \bar{\rho} \widetilde{u}_{i} \widetilde{u}_{j}}{\partial x_{j}}= & -\frac{\partial \bar{p}}{\partial x_{i}}+\frac{\partial}{\partial x_{j}}\left\{\mu\left(\frac{\partial \bar{u}_{i}}{\partial x_{j}}+\frac{\partial \bar{u}_{j}}{\partial x_{i}}-\delta_{i j} \frac{\partial \bar{u}_{k}}{\partial \bar{x}_{k}}\right)\right\} \\
& -\frac{\partial}{\partial x_{j}}\left\{\bar{\rho}\left({\widetilde{u_{i} u_{j}}}_{u_{i}} \widetilde{u}_{i} \widetilde{u}_{j}\right)\right\} \\
& +\bar{\psi}_{v} \bar{u}_{i, d}+\bar{\psi}_{i, m} \\
\frac{\partial \bar{\rho} \widetilde{Z}}{\partial t}+\frac{\partial \bar{\rho} \widetilde{Z} \widetilde{u}_{j}}{\partial x_{j}}= & \frac{\partial}{\partial x_{j}}\left\{\frac{\mu}{\operatorname{Sc}} \frac{\partial \bar{Z}}{\partial x_{j}}-\bar{\rho}\left(\widetilde{Z u_{j}}-\widetilde{Z}_{j}\right)\right\} \\
& +\left(\frac{v_{O}}{v_{O}+Y_{O}^{\max }}\right) \bar{\psi}_{v}
\end{aligned}
$$

where $Z$ is a mixture fraction, which is a scalar parameter representing the local concentration of a fuel or oxygen. The Schmidt numbers of all species are assumed to be the same for simplicity. $\psi_{v}$ is the vaporization source term, which represents the mass change of the droplets due to vaporization and couples the Eulerian (gas phase) and the Lagrangian (dispersed spray droplets) systems. To take into account the subgrid-scale effects on the resolved-scale motions, we adopt the Smagorinsky model:

$$
\begin{aligned}
& \bar{\rho}\left(\widetilde{u_{i} u_{j}}-\widetilde{u}_{i} \widetilde{u}_{j}\right) \equiv-2 \mu_{S G S} \bar{S}_{i j}, \\
& \mu_{S G S}=\bar{\rho}\left(C_{s} \Delta\right)^{2}|\bar{S}|, \\
& \bar{S}_{i j} \equiv \frac{1}{2}\left(\frac{\partial \bar{u}_{i}}{\partial x_{j}}+\frac{\partial \bar{u}_{j}}{\partial x_{i}}\right),
\end{aligned}
$$

where $|\bar{S}|$ is the norm of $\bar{S}_{i j}$. The model parameter $C_{S}$ (known as the Smagorinsky constant) is set to 0.1 . The subgrid-scale turbulent flux is modeled with the eddy diffusivity model:

$$
\bar{\rho}\left(\widetilde{Z u_{j}}-\widetilde{Z u_{j}}\right) \equiv-\frac{\mu_{S G S}}{\operatorname{Sc}_{S G S}} \frac{\partial \bar{Z}}{\partial x_{j}}
$$

where the turbulent Schmidt number, $\mathrm{Sc}_{S G S}$, is set to 0.5.

\subsection{Governing Equations for Droplet Motion}

The spray droplets are described using a Lagrangian technique, in which the droplets are tracked by Lagrangian particles translating in the Eulerian gas field. The physical properties of the droplets are estimated by solving the following equations for each droplet:

$$
\begin{aligned}
& \frac{d x_{i, d}^{k}}{d t}=u_{i, d}^{k}, \\
& \frac{d\left(D_{d}^{k}\right)^{3}}{d t}=-\frac{6}{\pi \rho_{d}} \dot{m}, \\
& \frac{d\left(D_{d}^{k}\right)^{3} u_{i, d}^{k}}{d t}=g \delta_{i 3}\left(D_{d}^{k}\right)^{3}+\frac{6}{\pi \rho_{d}}\left\{F_{i}^{k}-\dot{m} u_{i, d}^{k}\right\},
\end{aligned}
$$

where $x_{i}^{k}, u_{i}^{k}, D_{d}^{k}, \rho_{d}$, and $\dot{m}$ are the position, velocity, diameter, density, and vaporization rate, respectively. Superscript $k$ indicates the droplet number, and subscript $d$ indicates physical values for the droplets. The drag force $F_{i}^{k}$, which depends on the Reynolds number based on the slip velocity between the droplet and the surrounding gas phase, is estimated by:

$$
\begin{aligned}
F_{i}^{k} & =-\frac{\pi}{8} C_{D} \operatorname{Re}_{d} \mu D_{d}^{k}\left(u_{i, d}^{k}-\widehat{u}_{i, g}\right) \\
\operatorname{Re}_{d} & =\frac{D_{d}^{k} \rho_{g}\left|u_{i, d}-\widehat{u}_{i, g}\right|}{\mu} \\
C_{D 0} & =\frac{24}{\operatorname{Re}_{d}}\left(1+0.15 \operatorname{Re}_{d}^{0.687}\right)
\end{aligned}
$$


where $\mathrm{Re}_{d}$ is the droplet Reynolds number, and $C_{D 0}$ is the drag coefficient. The math operator $(\cdot)$ means the physical value at the corresponding droplet location estimated using tri-linear interpolation.

The following equation is the energy equation for each droplet.

$$
c_{p, d} m_{d}^{k} \frac{d T_{d}^{k}}{d t}=\pi h\left(D_{d}^{k}\right)^{2}\left(\widehat{T}_{g}-T_{d}^{k}\right)-\dot{m}^{k} L_{v},
$$

where $m_{d}$ and $L_{v}$ are the mass of the droplet and latent heat of vaporization, respectively. The heat transfer coefficient $h$ is evaluated by using the Ranz \& Marshall correlation ${ }^{(14)}$, as follows:

$$
\mathrm{Nu} \equiv \frac{h D_{d}}{\lambda}=2.0+0.6 \operatorname{Re}^{1 / 2} \operatorname{Pr}^{1 / 3},
$$

where $\mathrm{Nu}$ is the Nusselt number and $\lambda$ is the thermal conductivity.

In this study, droplet vaporization was simulated by means of the single-droplet and quasi-steady model $^{(1)}$, as follows:

$$
\dot{m}^{k}=\pi D_{d}^{k} \rho_{g} \frac{\mu}{\mathrm{Sc}} \operatorname{Sh} \ln \left(1+B_{M}\right)
$$

The Sherwood number Sh is calculated by semi-empirical equation proposed by Fröslling ${ }^{(15)}$ :

$$
\mathrm{Sh}=2+0.552 \mathrm{Re}^{1 / 2} \mathrm{Sc}^{1 / 3}
$$

where $B_{M}$ is the mass transfer number defined as follows:

$$
B_{M}=\frac{Y_{f}^{s}-Y_{f}^{\infty}}{1-Y_{f}^{s}},
$$

where $Y_{f}^{s}$ denotes the fuel mass fraction on the droplet surface, and $Y_{f}^{\infty}$ is the fuel mass fraction at the surrounding gas phase.

It is well known that drag reduction occurs due to droplet vaporization. We employed the model by Eisenklam ${ }^{(16)}$ to represent this drag reduction effect, as follows:

$$
C_{D}=C_{D 0} \frac{1}{1+B_{M}}
$$

The source terms in Eqs. (4)-(6) are estimated in the following equations:

$$
\begin{aligned}
& \bar{\psi}_{v}=\frac{1}{V} \sum_{k=1}^{n} \dot{m}^{k}, \\
& \bar{\psi}_{i, v}=\frac{1}{V} \sum_{k=1}^{n} \dot{m}^{k} u_{i, d}^{k}, \\
& \bar{\psi}_{i, m}=\frac{1}{V} \sum_{k=1}^{n} \psi_{i}^{k},
\end{aligned}
$$

where $\psi_{v}, \psi_{i, v}$, and $\psi_{i, m}$, are the contribution of the mass of the vaporized fuel gas, momentum of the vaporized fuel gas, and inertial force of each droplet, respectively. Additionally, $V$ is the volume of a defined computational cell, and $n$ is the total number of droplets.

The combustion gas temperature can be obtained from a flamelet library when employing the flamelet model for combustion modeling.

However, in the case of turbulent spray combustion, there is thermal interaction between combustion gas and spray droplets. Therefore, the flamelet model can not incorporate such influences into the flame temperature.

To take into account inter-phase coupling between the combustion gas and spray droplets, a simple thermal energy coupling model is proposed as follows:

$$
\widetilde{T}=T_{0}+c_{p, g} \rho_{g} \sum^{k}\left\{\frac{\pi \lambda D_{d}^{k}}{c_{p, d} m_{d}^{k}} \operatorname{Nu}\left(\widehat{T}_{g}-T_{d}^{k}\right)\right\},
$$


where $T_{0}$ is the preliminary temperature obtained from the flamelet library. The second term on the right-hand side corresponds to convection source terms in Eq. (17).

Inter-droplet physics such as collision, break-up, and coalescence are neglected in the present calculation because the Weber number for each droplet is very low and a dilute spray is considered. An influence of the gas-phase subgrid-scale fluctuation on the droplet motion is evaluated by a random walk model under the assumption that the gas-phase subgrid-scale fluctuation is isotropic and follows Gaussian distribution.

\subsection{Numerical Methods and Conditions}

The Favre-filtered momentum equations and the mixture fraction transport equation are solved using a finite-difference scheme in a cylindrical coordinate system.

We applied the second-order central differencing to the convection and the diffusion terms of the mass and momentum equations, and we used the third-order Runge-Kutta method (17) for time integration. The QUICK scheme, an upwind method, is adapted to the convection term in the scalar (mixture fraction) transport equation to maintain numerical stability. For pressure coupling, the fractional step method is used.

The flamelet equations were solved a priori using CHEMKIN ver. 3, while kinetic mechanism for methanol ${ }^{(18)}$ was used to obtain the flamelet library.

The reduced chemical kinetic mechanism ${ }^{(18)}$ used in the present work that involves 16 chemical species and 31 reactions.

We used a $\beta$ function to describe the sub-grid mixture fraction variance ${ }^{(19),(20)}$. To estimate the sub-grid scale mixture fraction variance, we presumed a local equilibrium and a scale similarity. The model for the variance can then be written as

$$
\widetilde{Z^{\prime \prime 2}}=c_{z}(\widetilde{Z}-\check{Z})^{2}
$$

where $\check{Z}$ is the test- and Favre-filtered mixture fraction, and a local constant $c_{z}$ is set to unity.

The Reynolds number, based on the inlet bulk air-flow velocity and burner-tip diameter, was set to approximately 21,000 (the same as the number used in the reference experiment described later). The number of grid points was $66 \times 37 \times 301$ in the radial, tangential, and axial directions, respectively. Twenty radial grid points were used to cover the inlet nozzle section. The inlet air flow velocity distribution was given by the measurement performed in previously ${ }^{(21)}$, as described below. We used the free-slip boundary condition on the lateral boundary and the convective condition at the outlet boundary.

The governing equations for the droplets were solved using the fourth-order Runge-Kutta scheme. The fuel was assumed to be methanol, and to be injected as individual droplets. The number of computational parcels was approximately 60,000 in fully developed combustion field. In this computation, we assumed that a droplet is fully vaporized when its diameter becomes smaller than 1 micron. The droplet size distribution was assumed to follow the conventional Nukiyama-Tanasawa equation. The coefficients in this equation were adjusted so that the Sauter mean diameter $D_{32}$ and arithmetic mean diameter $D_{10}$ matched the experimental results.

\section{Reference Experiment}

An experimental model of spray combustor ${ }^{(21)}$ was chosen to validate the present numerical study. In this experiment, methanol fuel spray and an axisymmetric turbulent air flow mixture were ignited and the combustion gas and the unburned spray droplets were discharged to an open domain through a 12.7-mm-diameter burner tip. The discharged gas velocity was around $25 \mathrm{~m} / \mathrm{s}$, and the droplet velocity was around $20 \mathrm{~m} / \mathrm{s}$. The Reynolds number, based on the burner-tip diameter and bulk gas velocity, was 21,000 . The mass-based overall equivalence ratio $\phi$ was 0.35 in this experiment.

The combustion gas temperature was measured using a spontaneous Raman spectroscopy technique, while phase Doppler anemometry (PDA) was used to obtain gas and spray veloc- 
ities. These measurements were performed at $z=0.1 D, z=0.5 D$, and at every half a burner diameter (D) thereafter, up to $z=6.5 \mathrm{D}$.

\section{Numerical Results}

Figure 1 shows the typical snapshot of the instantaneous numerical results. In spite of homogeneous injection of spray droplets in time, we observed dense or dilute structure of spray droplets invoked by the influence of the local vortical structure.

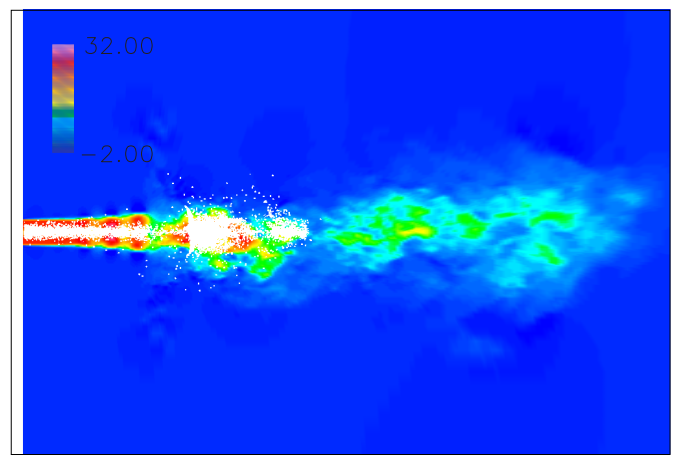

Fig. 1 Typical snapshot of the instantaneous numerical results (velocity contour plot and individual droplets)

The statistical averaged streamwise velocity component of the gas and dispersed phases on the central axis compared with the experimental data are shown in Fig. 2. The spray droplets acceleration by the surrounding air flow at an early stage of injection is in great agreement with the experimental data. Although the numerical results is slightly fluctuated compare with the experimental results, this is not affected the overall tendency of these results mentioned above.

This result derived by drag and vaporization effect is modeled appropriately. According to the regime diagram by Elghobashi ${ }^{(13)}$, the interaction between particle motion and turbulent motion under the present conditions is classified as a "two-way coupling" regime. Therefore, we can say that the inter-phase coupling obtained by two-way coupling using GS components is considered reasonable and proper. The spray velocity profile shows the same trend as the gas velocity profile further downstream. The gas and dispersed phases interaction phenomenon in this region is classified as a "one-way coupling regime", the numerical and experimental results are also in good agreement as a natural consequence.

Figure 3 shows the statistical averaged temperature distribution at various axial locations. In this figure, the gas moves downstream, while the temperature decreases rapidly at axial locations farthest from the burner exit $(6.5 D)$. This event corresponds to the flame front of the

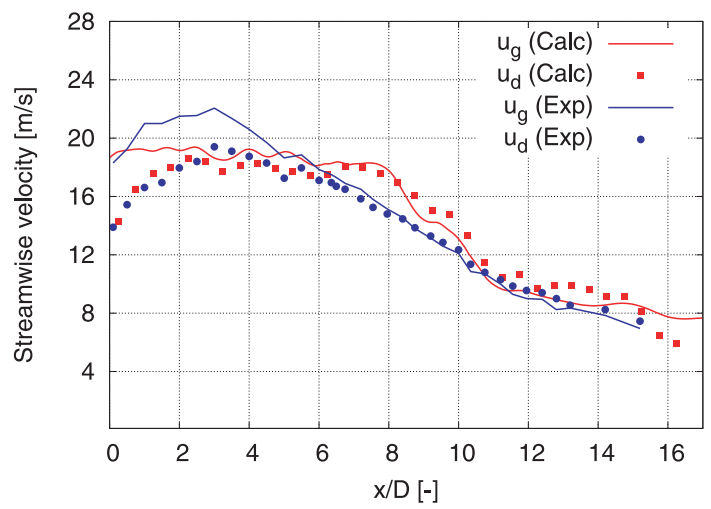

Fig. 2 Axial velocity components of the gas and dispersed phases at the central axis 


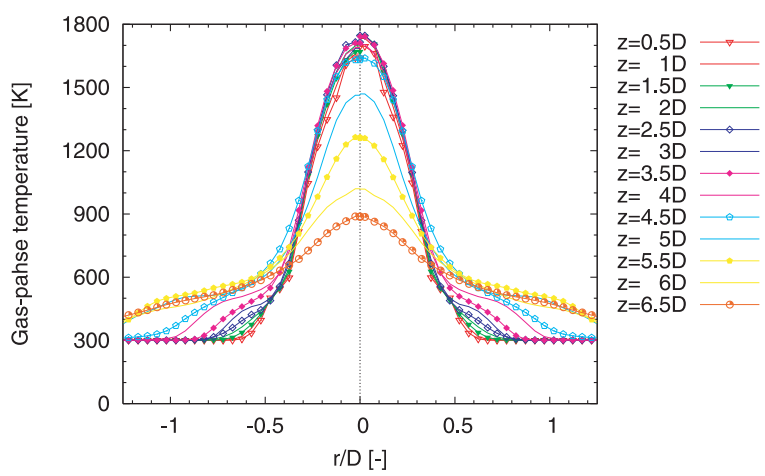

Fig. 3 Temperature distributions at various axial locations (left: with the thermal energy coupling model, right: without the coupling)

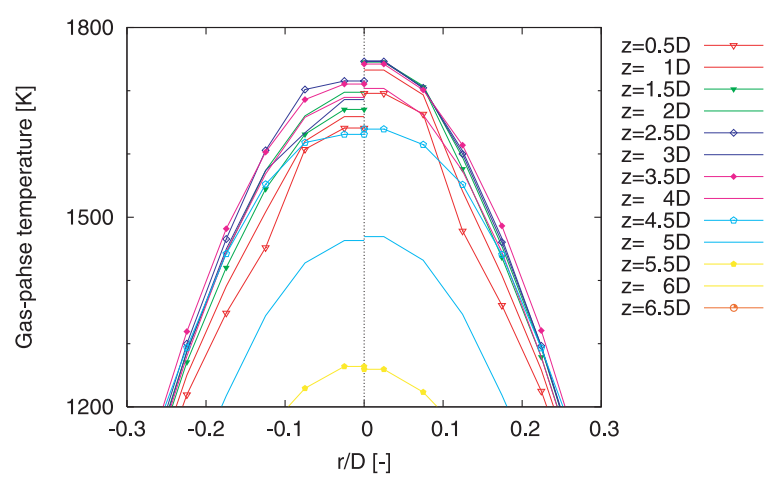

Fig. 4 Detailed view near the central axis of Fig. 3

spray flame and shows the same trend as that reported in the experiment ${ }^{(21)}$.

To validate the influence of the thermal energy coupling model (Eq. (26)) described in the previous section, we provide the result with the thermal energy coupling effect on the lefthand side and the result without the thermal energy coupling on the right-hand side of Fig. 3, and Fig. 4 is the detailed view of Fig. 3 near the central axis. These figures indicate that the burnt gas near the central axis is cooled down because of the dense existence of spray droplets. In the downstream region, on the other hand, this influence is decreased because of the sparse dispersion of the liquid spray droplets.

The maximum flame temperature is around $1,600 \mathrm{~K}$ in experiment ${ }^{(21)}$, on the other hand, $1,720 \mathrm{~K}$ is observed in this study. The tendency that the numerical results indicated higher temperature compare with the experimental results is still remain. However, the prediction is improved when the energy coupling between the gas and dispersed phases proposed by this study is taken into account.

\section{Conclusions}

LES solver which can be used to determine turbulent spray combustion flow accurately, has been developed in conjunction with the Eulerian/Lagrangian method for gas phase and dispersed phase representation and the flamelet approach for combustion modeling. In this procedure, we proposed a new thermal energy coupling model to estimate the thermal interaction between the gas phase and the dispersed phase.

We applied the numerical procedure to a laboratory-sized spray combustor and compared the numerical results for the axial velocity component of the individual spray droplets with the experimental results. We observed that these results show good agreement with experimental results and also confirmed that the inter-phase coupling for the mass and momentum was represented by the two-way coupling of GS components. This representation was confirmed 
by the regime diagram by Elghobashi ${ }^{(13)}$. The flame temperature drop caused by the thermal impact from the spray droplets was expressed properly by employing the thermal coupling model proposed in this study.

Consequently, using the numerical procedure based on LES proposed in this study, we have been able to predict appropriately the key features of turbulent spray combustion phenomena, such as the temperature distribution of the combustion gas, individual droplet behavior, droplet vaporization phenomena, and momentum interaction between the gas and dispersed phases.

\section{References}

( 1 ) A. D. Gosman, and E. Ioannides, "Aspects of Computer Simulation of Liquid-Fueled Combustor”, J. Energy, Vol. 7, No. 6, pp. 482-490, (1983).

( 2 ) T. Takagi, et al. "Numerical Simulation of Evaporation, Ignition and Combustion of Transient Sprays”, Combust. Sci. and Tech., vol. 75, pp. 1-12, (1991).

( 3 ) K. Akselvoll, and P. Moin, "Large-eddy simulation of turbulent confined coannular jets", J. Fluid Mech., Vol. 315, pp. 387-411, (1996).

( 4 ) Q. Wang, and K. D. Squires, "Large eddy simulation of particle deposition in a vertical turbulent channel flow”, Int. J. of Multiphase Flow, Vol. 22, No. 4, pp. 667-683, (1996).

( 5 ) K. Lei, N. Taniguchi, and T. Kobayashi, "Full Way Coupling of Large Eddy Simulation for Particle-Laden Turbulent Flows using New Dynamic SGS Models", Procs. of Turbulence and Shear Flow Phenomena 2, Vol. 2, pp. 111-116, (2001).

( 6 ) H. Pitsch, and H. Steiner, "Large-eddy simulation of a turbulent piloted methane/air diffusion flame", Physics of Fluids, Vol. 12, No. 10, pp. 2541-2554, (2000).

( 7 ) H. Forkel, and J. Janicka, "Large-Eddy Simulation of a Turbulent Hydrogen Diffusion Flame", Flow, Turbulence and Combustion, Vol. 65, No. 2, pp. 163-175, (2000).

( 8 ) Y. Itoh, N. Taniguchi and T. Kobayashi, "Numerical Prediction of Vaporizing Spray by using Large Eddy Simulation in Swirling Flows", Computational Fluid Dynamics Journal, Vol. 13, No. 4, pp. 703-709, (2005).

( 9 ) R. Kurose, O. Desjardins, M. Nakamura, F. Akamatsu and H. Pitsch, "Numerical simulations of spray flames", Annual Research Briefs, Center for Turbulence Research, NASA Ames/Stanford University, pp. 269-280, (2004).

(10) N. Patel, and S. Menon, "Simulation of spray-turbulence-flame interactions in a lean direct injection combustor", Combust. and Flame Vol. 153, pp. 228-257, (2008).

(11) Y. Baba and R. Kurose, "Validity of flamelet/progress-variable approach on numerical simulation of spray combustion”, Trans. JSME (B), Vol. 73, pp. 863-870, (2007) (in Japanese).

(12) Y. Baba and R. Kurose, "Analysis and flamelet modeling for spray combustion", J. Fluid Mech., Vol. 612, pp. 45-79, (2008).

(13) S. Elghobashi, "On Predicting Particle-Laden Turbulent Flows", Applied Scientific Research, Vol. 52, pp. 309-329, (1994).

(14) W. E. Ranz and W. R. Marshall, Jr., "Evaporation from Drops Part I, II", Chem. Eng. Prog., Vol. 48, pp. 141-146, pp. 173-180, (1952).

(15) C. K. Law and F. A. Williams, "Kinetics and convection in the combustion of alkane droplets", Combust. and Flame, Vol. 48, pp. 393-405, (1972).

(16) P. Eisenklam, S. A. Runachalam, J. A. Weston, "Evaporation rates and drag resistance of burning drops”, ,Procs. of Combustion Institute, Vol. 11, pp. 715-728, (1967).

(17) M. Hirohata, N. Taniguchi and T. Kobayashi, "Large Eddy Simulation of Lifted NonPremixed Jet Flames Using 2-Scalar Flamelet Model”, Procs. of FEDSM '03, 4TH ASME-JSME Joint Fluids Eng. Conf., (2003).

(18) E. Gutheil, G. Balakrishnan and F. A. Williams, "Structure and Extinction of HydrogenAir Diffusion Flames, in: Reduced Kinetic Mechanisms for Application in Combustion Systems (N. Peters, B. Rogg, Eds.)", Springer, (1993). 
(19) N. Branley, and W. P. Jones, "Large Eddy Simulation of a Turbulent Non-premixed Flame", Combust. and Flame, Vol. 127, pp. 1914-1934, (2001).

(20) V. Raman and H. Pitsch, "Large-eddy simulation of a bluff-body-stabilized nonpremixed flame using a recursive filter-refinement procedure", Combust. and Flame, Vol. 142, pp. 329-347, (2005).

(21) A. N. Karpetis, and A. Gomez, "An Experimental Study of Well-Defined Turbulent Nonpremixed Spray Flame”, Combust. and Flame, Vol. 121, No. 1, pp. 1-23, (2000). 